\title{
Phonological Encoding Is Not Contingent on Semantic Feature Retrieval: An Electrophysiological Study on Object Naming
}

\author{
Rasha Abdel Rahman \\ Max Planck Institute for Psycholinguistics
}

\author{
Miranda van Turennout \\ Max Planck Institute for Psycholinguistics and F. C. Donders \\ Centre for Cognitive Neuroimaging
}

Willem J. M. Levelt

Max Planck Institute for Psycholinguistics

\begin{abstract}
In the present study, the authors examined with event-related brain potentials whether phonological encoding in picture naming is mediated by basic semantic feature retrieval or proceeds independently. In a manual 2-choice go/no-go task the choice response depended on a semantic classification (animal vs. object) and the execution decision was contingent on a classification of name phonology (vowel vs. consonant). The introduction of a semantic task mixing procedure allowed for selectively manipulating the speed of semantic feature retrieval. Serial and parallel models were tested on the basis of their differential predictions for the effect of this manipulation on the lateralized readiness potential and N200 component. The findings indicate that phonological code retrieval is not strictly contingent on prior basic semantic feature processing.
\end{abstract}

The ability to recognize and name objects from vision is a seemingly effortless, highly practiced everyday skill. For example, after seeing a big gray creature with large ears and a trunk we can easily produce the name elephant at high speed. Visual perception also triggers access to rich sources of semantic information stored in memory. Attributes like is an animal, is a herbivore, lives in Africa, Asia, or in the zoo become available as well. A crucial research question in speech production concerns how and when these processes contribute to the production of the word elephant. The present experiments were designed to examine, with electrophysiological measures, the role of semantic feature processing for phonological encoding in picture naming. More specifically, we investigated whether retrieval of the word form is mediated by semantic feature retrieval (serial architecture) or whether the extraction of semantic information and phonological encoding can proceed independently (parallel architecture).

Most models of speech production distinguish three core processes in mapping an idea onto a spoken word, and these operations are thought to have different time courses (e.g., Bock, 1996; Dell, 1986; Levelt, 1989; Levelt, Roelofs, \& Meyer, 1999). The three processes make available conceptual-semantic, syntactic, and

Rasha Abdel Rahman and Willem J. M. Levelt, Max Planck Institute for Psycholinguistics, Nijmegen, the Netherlands; Miranda van Turennout, Max Planck Institute for Psycholinguistics and F. C. Donders Centre for Cognitive Neuroimaging, Nijmegen, the Netherlands.

We appreciate the constructive suggestions and comments of Mike Coles and three anonymous reviewers on an earlier version of this article and thank Helga Harsay for assistance in data collection.

Correspondence concerning this article should be addressed to Rasha Abdel Rahman, Max Planck Institute for Psycholinguistics, P.O. Box 310, 6500 AH Nijmegen, the Netherlands. E-mail: rasha.abdel-rahman@mpi.nl phonological information, respectively. In a first step, the target conceptual representation is selected. This representation activates the appropriate lexical candidate, the lemma. Furthermore, the concept node spreads activation to related concepts that in turn activate their corresponding lexical candidates. The second step, then, is to select the target lemma from among coactivated lemmas. With lemma retrieval, syntactic features such as word class, syntactic gender, or number become available. In a third step, the phonological code corresponding to the selected lemma is retrieved (e.g., Levelt, 1989; Levelt et al., 1999). The retrieved phonological code, largely an ordered set of phonemic segments, is input to a set of phonological and phonetic operations that compute the ultimate articulatory motor program. Whether phonological codes of nontargets are occasionally coactivated is a matter of discussion (e.g., Dell, 1986; Dell \& O’Seaghdha, 1992; Jescheniak \& Schriefers, 1998; Peterson \& Savoy, 1998).

The aim of the present study was to test whether retrieval of a basic semantic feature, such as animacy, is conditional for lemma selection and hence occurs prior to phonological code retrieval. It is contrasted with a parallel account, in which lemma selection is not conditional on the retrieval of any specific semantic feature. Even though speech production models agree that for content words lemma selection is conceptually driven in nature, parallel retrieval of semantic features and word forms remains a theoretical option. For example, nondecompositional theories suggest that concepts are represented as undivided wholes (Collins \& Loftus, 1975; Fodor, 1976; Levelt et al., 1999; Roelofs, 1992, 1997). Each lemma node is linked to a single individual concept node from which it receives activation. Any distinct features representing the facets of word meaning are retrieved via labeled links to other concept nodes such as ELEPHANT is an ANIMAL (cf. Levelt et al., 1999). In principle, these theories of lemma retrieval allow for 
word-form encoding without prior access to a core variety of potentially retrievable semantic features stored in memory. Selection of the concept node suffices, and it is irrelevant from which semantic or perceptual feature the concept node received its activation. In other words, semantic retrieval can proceed in parallel to retrieval of the phonological code.

\section{The Lateralized Readiness Potential (LRP) in Two-Choice Go/No-Go Tasks}

Recently a number of studies used components of event-related brain potentials (ERPs) to investigate the time course of semantic, syntactic, and word-form encoding in picture naming (Schmitt, Münte, \& Kutas, 2000; Schmitt, Rodriguez-Fornells, Kutas, \& Münte, 2001; Schmitt, Schilz, Zaake, Kutas, \& Münte, 2001; van Turennout, Hagoort, \& Brown, 1997, 1998). In particular, van Turennout et al. $(1997,1998)$ introduced the LRP into the field of language production research. The LRP is extracted from the readiness potential (RP) that precedes voluntary hand movements (Kornhuber \& Deecke, 1965). Later portions of the RP develop asymmetrically over the left and right motor cortex as a function of the given response, being more negative over areas contralateral to the responding hand (Kutas \& Donchin, 1974, 1980; Vaughan, Costa, \& Ritter, 1968). This lateralized part of the RP can be used as an index for specific response preparation (Kutas \& Donchin, 1980). To isolate the lateralized part of the RP from all other brain potentials measured at the scalp, the LRP is computed with respect to the correct response hand (cf. Coles \& Gratton, 1986; De Jong, Wierda, Mulder, \& Mulder, 1988; Smid, Mulder, \& Mulder, 1987). First, for both left and right hand responses the potential recorded over ipsilateral motor areas is subtracted from the potential recorded over contralateral areas (cf. Coles, 1989). These waveforms are averaged separately for trials in which the right hand was cued and for trials in which the left hand was cued. Then, by averaging across left and right hand trials, all lateralized potentials that are not specifically related to response preparation are canceled out. The resulting LRP reflects the average amount of lateralization occurring as a result of central motor preparation.

The LRP is a useful tool in the study of cognitive processes (for a comprehensive review see Eimer \& Coles, 2003). Most important for the current study, it has been shown that in combination with a two-choice go/no-go paradigm, the LRP can be used to assess the nature of information transmission. In a study by Miller and Hackley (1992), participants classified a letter stimulus on two dimensions. The shape of the letter ( $S$ or $T$ ) determined response hand (left or right), and the size of the letter (small or large) specified whether the response was emitted (go trials) or withheld (no-go trials). With the occurrence of an LRP in trials in which the response was successfully withheld (no-go LRP), the authors showed that the rapidly available shape information was transmitted to motor stages for response preparation before the less salient size information was available to determine the response decision. Similar no-go LRP activity has been reported by Smid, Mulder, Mulder, and Brands (1992) when color determined the choice response and the go/no-go decision depended on letter identity and by Osman, Bashore, Coles, Donchin, and Meyer (1992) when the choice response was based on the spatial position of the stimulus whereas stimulus identity (letter vs. digit) specified the go/no-go decision. Together, the no-go LRP results show that response preparation can start on the basis of partial stimulus evaluation that is transmitted to motor stages before stimulus analysis has been completed.

\section{Electrophysiological Investigations of Speech Production}

The LRP's sensitivity to low levels of response activation was used by van Turennout et al. (1997) to assess the time course of semantic and word-form encoding in speech production. In a similar two-choice go/no-go task, pictures of objects were manually classified on the basis of a semantic feature (animacy) and on the basis of a phonological feature of the object name (e.g., word final $/ \mathrm{r} / \mathrm{vs}$. $/ \mathrm{n} /$ ). An LRP in no-go trials was present only when the response hand was specified by a semantic feature but not when it depended on phonology. These results show that in picture naming, semantic information is available earlier than is phonological information, and semantic knowledge can be transmitted to motor stages before name retrieval has been completed. Using a similar experimental paradigm, but with German stimulus materials and participants, Schmitt et al. (2000) replicated these LRP findings. In addition, they reported differential effects of semantic and phonological retrieval on response inhibition, as measured by the N200 on no-go trials. The N200 component involves a negativity over frontocentral sites, which is larger on no-go than on go trials and is presumed to be related to response inhibition (cf. Jodo \& Kayama, 1992; Simson, Vaughan, \& Ritter, 1977; Thorpe, Fize, \& Marlot, 1996; for an opposing view see Nieuwenhuis, Yeung, van den Wildenberg, \& Ridderinkhof, in press). The onset and peak latency of the N200 were computed by Schmitt et al. (2000) as estimates of the moments at which execution-relevant (go/no-go) information is available. They found that the N200 onset and peak latency was earlier when the go/no-go decision was based on the semantic classification than when it was based on the phonological classification. Again, these results show that in picture naming, information about animateness is available earlier than is phonological information. These results were interpreted as evidence for serial or cascaded models of semantic and name retrieval, in contrast to parallel processing. The serial model with the expected LRP and N200 time course is depicted in Figure 1.

However, these well-established differences in the time course of semantic and phonological retrieval do not necessarily imply that form encoding depends on prior semantic feature retrieval, as suggested by serial models. If the no-go LRP pattern would be taken as exhaustive evidence for serial and against parallel processing one would have to conclude that, for example, the analyses of letter identity depends on prior color processing (cf. Smid et al., 1992) or that the size of a letter stimulus cannot be established before its identity has been determined (Miller \& Hackley, 1992). Alternatively, accessing phonological information might simply be slower than retrieval of semantic features-without phonological encoding being contingent on prior semantic processing. Therefore, parallel or independent processing cannot be ruled out. Figure 2 presents the identical LRP and N200 time course within a parallel arrangement.

With the aim of distinguishing between serial and parallel retrieval, Abdel Rahman and Sommer (2003) used a similar twochoice go/no-go procedure and additionally manipulated the duration of semantic processing. The critical question was whether the duration of semantic processing affected the beginning of word- 
A

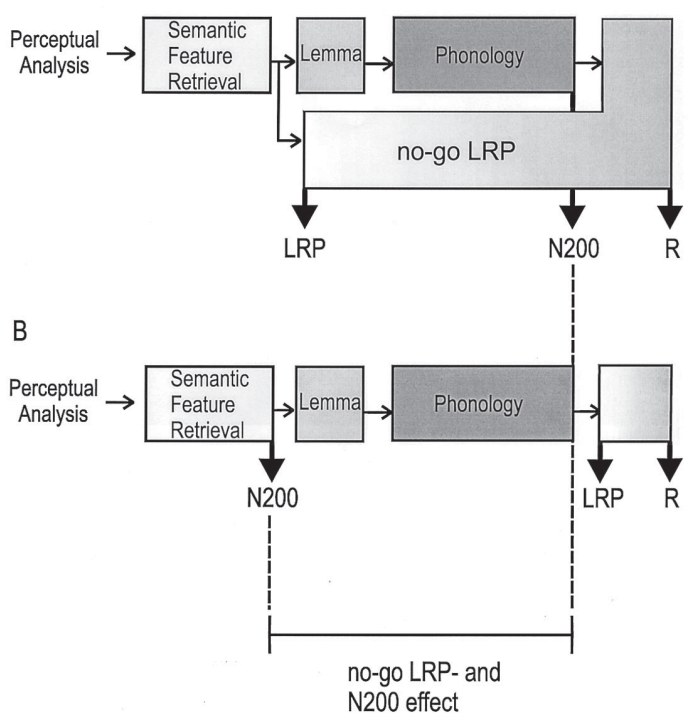

Figure 1. Schematic illustration of the serial model with the predicted no-go lateralized readiness potential (LRP) and N200 effects in two-choice go/no-go tasks. A: Response hand is based on a semantic classification and the go/no-go decision is based on a classification of name phonology. B: Response hand is based on a classification of name phonology and the go/no-go decision is based on a semantic classification. $\mathrm{R}=$ response.

form encoding, as predicted by serial models. In the first experiment, pictures of animals were classified according to their size in real life (small vs. large) or according to their diet (herbivorous vs. omnivorous). The semantic classification determined the response hand, whereas the go/no-go decision depended on a phonological classification (word initial vowel vs. consonant). Here, a no-go LRP was present only for the comparatively fast size classification but not for the more difficult and slow diet classification. Moreover, the interval between the terminations of semantic retrieval and word-form encoding decreased as the duration of semantic processing increased. To estimate the length of this interval for both semantic conditions, Abdel Rahman and Sommer computed LRPs time locked to the response in go trials. The response-locked LRP (R-LRP) results revealed that the interval between LRP onset and the response was shorter in the slow than in the fast semantic condition. This means that less time was required to execute the response after response hand selection in the slow semantic condition as compared with the fast semantic condition. The effects of both the stimulus-locked LRP (S-LRP) in no-go trials and the $\mathrm{R}-\mathrm{LRP}$ in go trials are in line with parallel models predicting that word-form encoding is not affected by the duration of semantic feature retrieval. In the second experiment, the choice response was based on name phonology, and the response decision depended on the fast (size) or slow (diet) classification; here, the LRP onset latency in go trials provides an upper estimate of the duration of name retrieval. As predicted by parallel models, and in contrast with serial models, semantic retrieval duration did not affect LRP onset latency in go trials.

Taken together, the reported LRP experiments reveal two lines of evidence. First, semantic features like animacy or size are available earlier than phonological information about the object name. Second, this does not necessarily imply serial ordering of these processes. A manipulation of semantic difficulty revealed that semantic retrieval does not, for any possible duration, precede word-form encoding. However, interpretation of these results in terms of parallel processing faces two main problems. First, two different semantic classification tasks have been used. One could argue that parallel retrieval holds for the difficult diet classification but not for the size classification. Although the empirical basis for such a distinction is weak, this alternative explanation cannot be ruled out on the basis of the given pattern of results. Second, even if retrieving features like size and diet is irrelevant for the onset of word-form encoding, there might be more basic semantic features which have to be available for one to name an object. So far, empirical evidence for such a suggestion is lacking. However, a promising candidate for an essential feature is animacy, because this feature has repeatedly been shown to be available faster than name phonology (Schmitt et al., 2000; van Turennout et al., 1997). Without attempting to make strong claims about a distinction between critical and noncritical semantic features for picture naming, we presumed animacy to be the most commonly accepted one. With our experiment we directly tested whether retrieval of this semantic feature is not only faster but also a necessary prerequisite for lemma selection and hence the onset of word-form encoding of the target word.

\section{Experimental Paradigm}

To distinguish between serial and parallel processing with the LRP in a two-choice go/no-go design, one must manipulate re-

A

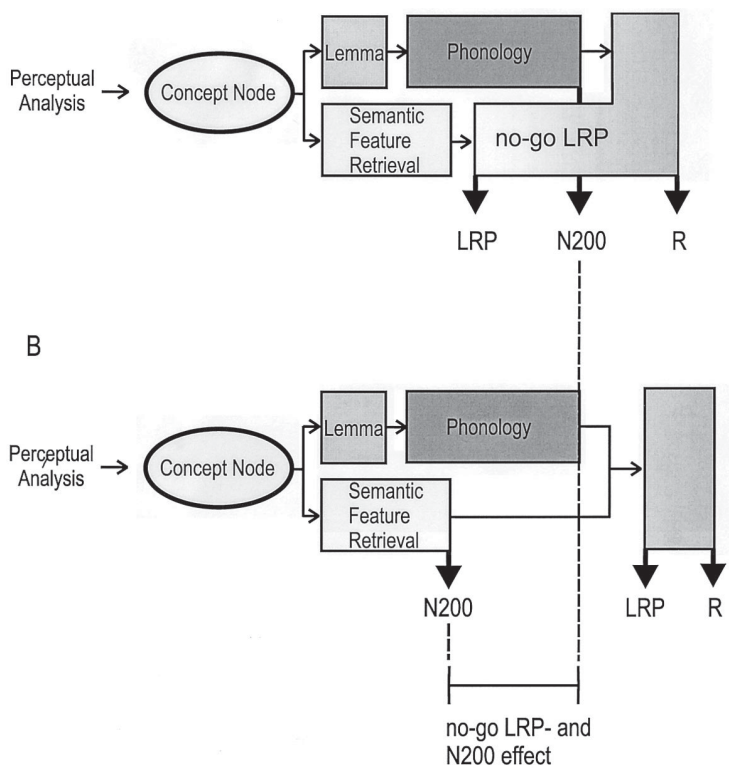

Figure 2. Schematic illustration of the parallel model with the predicted no-go lateralized readiness potential (LRP) and N200 effects in two-choice go/no-go tasks. A: Response hand is based on a semantic classification and the go/no-go decision is based on a classification of name phonology. B: Response hand is based on a classification of name phonology and the go/no-go decision is based on a semantic classification. $\mathrm{R}=$ response. 
trieval latency of the faster and presumably mediating (i.e., the semantic) process. This manipulation allows one to examine whether the beginning of phonological encoding is affected by the duration of semantic retrieval, as predicted by serial processing models. Because of the above-mentioned disadvantages of using two semantic classification tasks, we introduced a task-mixing procedure to selectively manipulate the duration of a single semantic classification. The basic semantic feature, animacy, was either repeatedly classified (blocked classification mode) or randomly alternated by an additional semantic classification (mixed classification mode). Task mixing has been shown to affect performance in various tasks (for a review see Los, 1996, 1999a, 1999b), and we expected it to slow down the animacy classification.

In the current experiment a two-choice go/no-go task was used in which the semantic classification specified the response hand and the phonological classification determined response execution. The experimental logic behind the manipulation of semantic processing speed is simple: If phonological code retrieval is mediated by semantic feature retrieval then it should be sensitive to the duration of semantic processing. In this case, the beginning of phonological encoding should vary as a function of semantic task mix. In contrast, if the two processes can proceed in parallel, the beginning of phonological encoding should not be affected by the speed of semantic processing. These differential predictions were tested with the S-LRP and R-LRP in go and no-go trials and in the N200 component, which are schematically depicted for the two alternative models in Figure 3.

\section{LRP Onset Latency}

Because the response hand is mapped to the semantic classification, the S-LRP onset latency in go trials provides an upper chronometric estimate of the speed of semantic processing. Therefore both serial and parallel models predict an effect in the S-LRP onset latency if semantic task mixing is an effective tool for controlling semantic processing speed.

\section{No-Go LRP}

In no-go trials both models predict early LRP activity (no-go LRP) at least in the fast semantic condition (blocked classification mode) because, as shown by van Turennout et al. (1997) and Schmitt et al. (2000), the animateness information is available faster than name phonology. The critical question here is whether a no-go LRP can also be observed in the mixed classification mode in which the animacy information is available less fast. According to serial models, name phonology can be extracted only after semantic information has been retrieved. Therefore, these models predict a no-go LRP regardless of semantic processing durationand hence also in the mixed-classification mode. In contrast, if semantic features and name phonology are processed in parallel, the presence of a no-go LRP depends on the relative duration of semantic and phonological encoding. If the semantic classification is slowed in the mixed classification mode, the no-go LRP activity should be largely reduced or even absent.

\section{$R-$ LRP Onset Latency}

The R-LRP in go trials provides an estimate of the time interval between the terminations of semantic and phonological encoding.
A

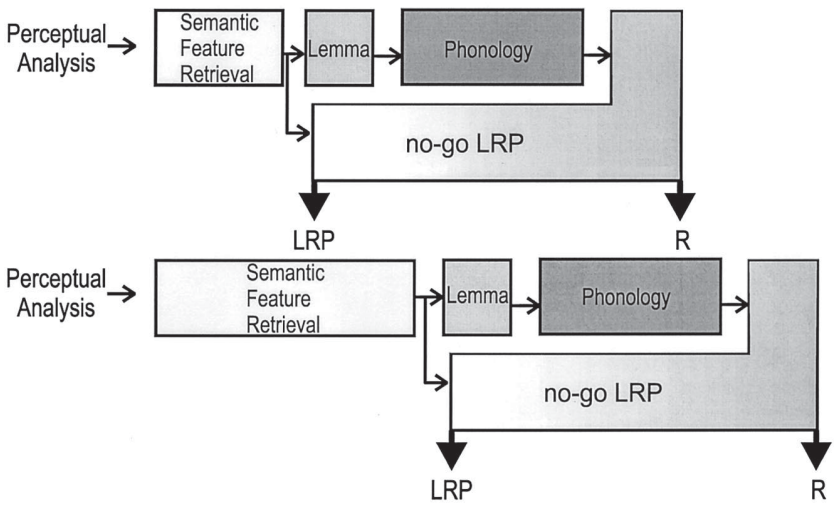

B

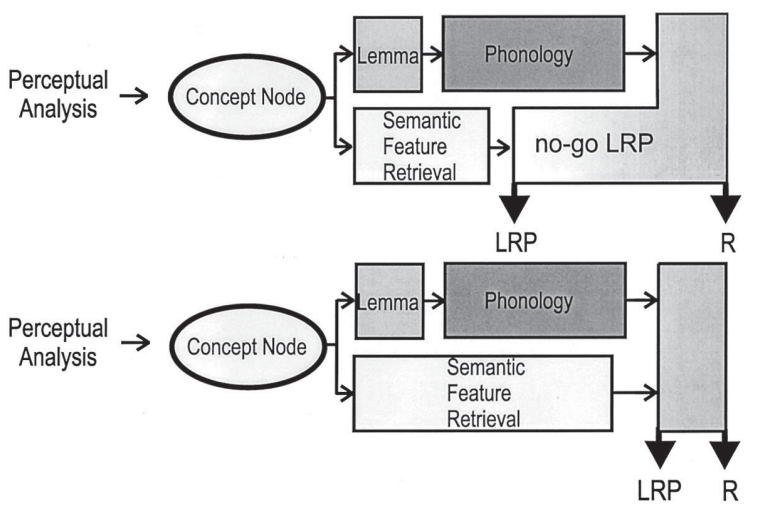

Figure 3. Differential predictions of serial (A) and parallel (B) models for the effects of semantic task mixing on the lateralized readiness potential (LRP) and N200. The beginning of central response activation (LRP onset) is contingent on the retrieval of semantic information; response inhibition (indicated by the N200) is contingent on name retrieval. A: Serial models predict a no-go LRP in both the blocked (fast) and the mixed (slow) semantic classification mode. The LRPresponse interval should not be affected by semantic task mix. N200 latency should vary as a function of semantic task mix. B: Parallel models predict a higher probability for the occurrence of a no-go LRP in the blocked, compared to the mixed, semantic classification mode. The LRP-response interval should be decreased in the mixed condition. No effect is expected for the $\mathrm{N} 200$ latency. $\mathrm{R}=$ response.

According to serial models this interval should comprise the entire duration of phonological encoding after the semantic feature has been retrieved. Because the experimental manipulation is located prior to phonological encoding, the R-LRP onset latency should not be affected by semantic processing duration. On the other hand, parallel models predict that the interval between the terminations of semantic and phonological encoding should decrease as the duration of semantic processing increases. Accordingly, the LRP-to-response interval should be shorter in the mixed, as compared with the blocked, classification mode.

\section{N200 Peak Latency}

Finally, the N200 was assumed to provide an upper estimate of the point in time when enough phonological information is avail- 
able for response inhibition. If phonological encoding is semantically mediated, the peak latency of the N200 should vary as a function of semantic processing duration. In contrast, no effect on the N200 was predicted if phonological encoding is not contingent on prior semantic feature retrieval.

\section{Preexperiment}

A preexperiment with simple choice responses was conducted to assess whether the duration of the animacy classification can be selectively manipulated with a task-mixing procedure. If the effect of semantic task mixing is confined to semantic classifications alone and does not result in a general slowing of performance, naming latencies and manual name-classification latencies should not be affected.

\section{Method}

Participants. Twenty-four native Dutch speakers from the participants pool of the Max Planck Institute were paid for taking part in the experiment. All participants had normal or corrected-to-normal visual acuity and normal color vision. The participants were randomly assigned to two experimental groups. Group 1 performed simple choice responses on two semantic dimensions (animacy: animal vs. object; location: typically found inside the water vs. typically found outside the water) interspersed with overt naming trials; Group 2 performed simple choice responses on the two semantic dimensions and on name phonology (second phoneme: vowel vs. consonant)

Material. The stimulus set (see the Appendix) consisted of 56 black line drawings of common objects (approximately $3.5 \mathrm{~cm} \times 3.5 \mathrm{~cm}$ ). The pictures were equally assigned to binary categories of the three orthogonal dimensions: animacy, location, and name phonology. For example, (a) the fox (Vos, in Dutch) is an animal, it lives outside the water, and the second phoneme of the word is a vowel or (b) the anchor (Anker, in Dutch) is an object, it is typically found in the water, and the second phoneme of the word is a consonant.

Procedure and design. Each trial started with the presentation of a black fixation cross in the middle of a light gray screen. After $500 \mathrm{~ms}$ the fixation cross was replaced by a framed target picture. In manual classification trials the picture was shown for $1.3 \mathrm{~s}$ with an interstimulus interval (ISI) of $1.8 \mathrm{~s}$. In naming trials the picture remained on the screen for $2 \mathrm{~s}$, resulting in an ISI of $2.5 \mathrm{~s}$. Responses for the manual classifications were recorded with response keys; voice onset latencies for naming responses were collected with a voice key trigger. Both latencies were measured during the entire period of picture presentation. The type of response ([a] naming or [b] manual classification of name phonology, animacy, or location) was indicated by frames in different colors that surrounded the pictures. Red frames indicated an animal versus object classification, blue frames indicated an inside-the-water versus an outside-the-water classification, and black frames indicated naming trials in Group 1 and name classification trials in Group 2. The location classification served as a means to induce mixing costs on the animacy classification. Participants were instructed to name or classify the pictures as fast and as accurately as possible. The experiment started with a practice block. Then, four experimental blocks of 168 trials each were carried out, subdivided by short breaks. In the blocked condition, a single type of semantic classification was performed within a block, whereas in the mixed condition, both semantic classifications were carried out in random alternation. In both conditions, the semantic classification trials were intermediated by one third of naming trials (Group 1) or name classification trials (Group 2). The order of block types and stimulus-response assignment for the manual classifications was counterbalanced across participants.

\section{Results and Discussion}

Separately for the two experimental groups, mean reaction times (RTs) and error rates (see Table 1) were submitted to Huynh-Feldt corrected repeated measures analyses of variance (ANOVAs) with the two-level variables of response type (animacy classification and naming or name classification, respectively) and task mix (blocked or mixed semantic classification). For RT analyses, trials with errors (wrong keypresses, omissions, incorrect naming, or nonspeech sounds preceding picture naming) and trials with latencies larger than $1.3 \mathrm{~s}$ for manual classifications and larger than $2 \mathrm{~s}$ for naming were discarded. In both groups, ANOVAs yielded main effects of response type, $F(1,11)=45.3, p<.01$, for Group $1 ; F(1,11)=162.1, p<.01$, for Group 2, and task mix, $F(1$, $11)=20.1, p<.01$, for Group $1 ; F(1,11)=17.1, p<.01$, for Group 2, and a Response Type $\times$ Task Mix interaction, $F(1$, $11)=53.9, p<.01$, in Group 1 , and $F(1,11)=9.5, p<.01$, in Group 2. Separate comparisons revealed that the main effect of task mix is carried by a strong effect on the semantic classification. Whereas semantic classification times were much larger in the mixed as compared with the blocked condition, mean difference $=$ $154 \mathrm{~ms}, t(11)=6.2, p<.01$, for Group 1 ; mean difference $=86$ ms, $t(11)=5.2, p<.01$, for Group 2; no such mixing costs were observed for picture-naming latencies, mean difference $=-9 \mathrm{~ms}$, $t(11)=0.7, p=.48$, or name classification latencies, mean difference $=28 \mathrm{~ms}, t(11)=1.6, p=.11$. In Group 1, there were no main effects of response type, $F(1,11)=2.0, p=.18$, and task $\operatorname{mix} F(1,11)=1.9, p=.18$, in error rates; in Group 2 there was a significant main effect of response type, $F(1,11)=56.9, p<$ .01 , but no effect of task mix, $F(1,11)=0.8$ (see Table 1 ).

The differential RT effect that task mix yielded on the semantic classification and naming in Group 1 and name classification in Group 2 confirms that semantic feature retrieval can be selectively manipulated by introducing an intervening semantic classification. Because the semantic classification was affected but the name classification or overt naming latency was not, task mixing at the semantic level does not seem to result in a general slowing of performance or, more specifically, to influence the duration of name retrieval.

Table 1

Mean Reaction Times (RTs) and Error Rates in the Preexperiment

\begin{tabular}{|c|c|c|c|c|}
\hline \multirow[b]{3}{*}{ Group } & \multicolumn{4}{|c|}{ Response type } \\
\hline & \multicolumn{2}{|c|}{ Animacy classification } & \multicolumn{2}{|c|}{ Naming } \\
\hline & $\mathrm{RT}(\mathrm{ms})$ & Error $(\%)$ & $\mathrm{RT}(\mathrm{ms})$ & Error $(\%)$ \\
\hline \multicolumn{5}{|c|}{ Blocked classification mode } \\
\hline 1 & 700 & 5.8 & 934 & 6.9 \\
\hline 2 & 791 & 7.6 & 997 & 24.2 \\
\hline \multicolumn{5}{|c|}{ Mixed classification mode } \\
\hline 1 & 854 & 10.4 & 925 & 4.7 \\
\hline 2 & 877 & 12.1 & 1,025 & 25.3 \\
\hline
\end{tabular}




\section{Main Experiment}

\section{Method}

Participants. Twenty-four native Dutch speakers (18-27 years of age, $M=25 ; 17$ women, 7 men) were paid for their participation in the experiment. None of the participants took part in the preexperiment. All participants were right-handed (mean handedness score $>70$; Oldfield, 1971) and reported normal or corrected-to-normal visual acuity and normal color vision. Eight participants were excluded and replaced because of excessive artifacts mostly due to whispering in silent naming trials.

Procedure. Stimuli included the 56 line drawings that were used in the preexperiments. Prior to the experiment all pictures were shown on a computer screen in random order, and participants were asked to name the pictures. After all pictures had been named, they were shown again, and participants were instructed to classify verbally whether an animal or object was depicted, whether it is typically found inside or outside of the water, and whether the second phoneme of the name was a vowel or consonant. After participants had successfully named all pictures and the relevant attributes, the experimental session started with two practice blocks with a blocked and mixed semantic classification mode, followed by eight experimental blocks with a total of 1,216 trials. The blocks were subdivided by short breaks. Participants were instructed to select the response hand on the basis of an animacy classification when the picture was presented in a red frame or on the basis of a location classification when the picture was presented in a blue frame. The go/no-go decision was always based on a classification of the second phoneme. In one third of the trials the pictures had to be named silently, which was indicated by a black frame surrounding the picture. These trials were introduced as a precaution against automatic stimulus-response mappings and to maintain phonological code retrieval. A trial began with the presentation of a fixation cross in the middle of the screen. After $500 \mathrm{~ms}$ the picture was presented for $1.5 \mathrm{~s}$ in two-choice go/no-go trials with an ISI of $2 \mathrm{~s}$ and for $2 \mathrm{~s}$ in silent naming trials with an ISI of $2.5 \mathrm{~s}$. Participants received feedback, shown for $300 \mathrm{~ms}$ on the screen, after wrong keypresses and misses in go trials and after false alarms in no-go trials. Because response preparation can be encouraged by a higher proportion of go relative to no-go trials (Low \& Miller, 1999), 16 pictures of the stimulus set that produced the highest error rates in the preexperiments were assigned to go-filler trials and excluded from data analyses (see the Appendix). Responses were recorded with response keys mounted behind each other in the midsagittal plane of the participant to avoid lateralized eye movements toward the response hand side. Participants were instructed to respond as quickly and accurately as possible and to keep their gaze on the fixation point or picture in the middle of the screen. Additionally, they were instructed not to blink during picture presentation to minimize electroencephalogram (EEG) artifacts because of eye movements. The assignment of the go and no-go decision to the name attribute was alternated blockwise. The order of alternations, order of semantic conditions (blocked and mixed semantic classification mode), and the assignment of semantic features to push-button responses was counterbalanced across participants.

Electrophysiological recordings. The EEG was recorded continuously with a $250 \mathrm{~Hz}$ sample rate at $\mathrm{Fz}, \mathrm{Fcz}, \mathrm{Cz}, \mathrm{Pz}$, and $\mathrm{Oz}$ as defined by the international 10-20 system (Jasper, 1958) and at the electrode sites C3' and $\mathrm{C}^{\prime}, 4 \mathrm{~cm}$ to the left and right of $\mathrm{Cz}$, respectively. Bandpass was set to $0.01-40.00 \mathrm{~Hz}$. The horizontal electrooculogram (hEOG) and vertical electrooculogram (vEOG) were recorded bipolarly from the external canthi and from above and below the midpoint of the right eye. To detect peripheral response activation in no-go trials the ectromyogram (EMG) was recorded from standard electrode placements for finger flexor muscles at the forearms (Lippold, 1967). All electrodes were referenced to the left mastoid. $\mathrm{Ag} / \mathrm{AgCl}$ electrodes were used and electrode impedance was kept below $5 \mathrm{k} \Omega$ for cephalic and face sites and below $10 \mathrm{k} \Omega$ for the forearm electrodes.
Data analyses. Trials with incorrect responses, ocular artifacts (blink threshold: $30 \mu \mathrm{V}$ ), or activity in any cephalic channel that exceeded $70 \mu \mathrm{V}$ and trials with an RT less than $100 \mathrm{~ms}$ were discarded from the analyses. Offline, the EEG was intersected into epochs of $1,800 \mathrm{~ms}$ around stimuli and responses. Stimulus-synchronized epochs began $200 \mathrm{~ms}$ prior to stimulus onset and were baseline adjusted by subtracting the average voltage during a prestimulus interval of $200 \mathrm{~ms}$. Response-synchronized epochs began 1,600 ms prior to RT and were baseline adjusted by subtracting the average voltage during an interval of 1,300-1,100 ms before the response. The LRP was computed separately for go and no-go trials for the two experimental conditions as the difference between the electrodes over the primary motor cortices $\left(\mathrm{C}^{\prime}\right.$ and $\left.\mathrm{C}^{\prime}\right)$ contra- and ipsilateral to the correct response hand. The difference waves were averaged first within and then across cued left- and right-hand responses (see the introduction). The hEOG and the EMG were computed analogously to assess possible contributions of lateralized eye movements on the LRP and to inspect no-go trials for peripheral activity, respectively. Latency differences for go LRPs between conditions were statistically analyzed with a jackknifing procedure that allows for paired comparisons (here one-tailed $t$ tests) between conditions for grand mean waveshapes rather than for individual curves (Miller, Patterson, \& Ulrich, 1998). Criteria for S-LRP and R-LRP onsets were $0.4 \mu \mathrm{V}$ and $0.8 \mu \mathrm{V}$ below baseline. The presence of LRP activity in no-go trials was assessed with two-tailed $t$ tests against zero for consecutive 20-ms time windows, starting $100 \mathrm{~ms}$ after stimulus onset. A no-go LRP was defined to be present if at least three successive intervals significantly differed from zero $(p<.05)$. The N200 was calculated as a difference wave between no-go and go trials for the two experimental conditions at the Fz electrode site. The vEOG was computed analogously, to assess possible contributions of vertical eye movements on the N200. The N200 latency was measured as the time point of the minimum in the waveform between 100 and $800 \mathrm{~ms}$ after stimulus onset. Latency differences of N200 peak latencies between conditions were assessed statistically with the jackknife procedure as described above.

\section{Results and Discussion}

Performance. RTs in go trials were shorter in the blocked than in the mixed semantic classification mode $(M=927 \mathrm{~ms}$ vs. $M=1,068 \mathrm{~ms}), t(23)=-6.8, p<.01$. An additional analyses of sequential effects within the mixed classification mode for animacy classification repetitions versus alternations $(M=1,025 \mathrm{~ms}$ vs. $M=1,122 \mathrm{~ms}$ ) yielded a similar pattern, $t(23)=-5.9, p<$ .01. Participants were faster in performing the animacy classification repeatedly than they were when the animacy classification was preceded by, or intermixed with, a different semantic classification. Mean error rate was lower in the blocked than in the mixed classification mode $(M=9.4 \%$ vs. $16.8 \%), t(23)=-5.1$, $p<.01$.

One might ask whether the RT effect is not in itself diagnostic with respect to the distinction between seriality and parallelity. Serial models as depicted in Figure 3 predict similar RT effects of semantic task mix in the single- and two-choice go/no-go condition, whereas parallel models predict an absent or at least reduced effect of semantic task mix in the two-choice go/no-go condition because the interval between the terminations of semantic and phonological processing should wash out (i.e., absorb) at least some proportion of the effect (see Figure 3B). However, RT effects cannot be taken as evidence distinguishing between the two types of models for at least one reason: According to parallel models, responses are expected to be activated in advance only in the blocked but not (or considerably less extensively) in the mixed classification mode. This advance motor preparation in the fast 
semantic condition should yield a temporal advantage as compared with the slower semantic condition in which responses are less likely to be activated in parallel to phonological encoding. As we show below, the selective occurrence of a no-go LRP is in line with an explanation of RT effects in terms of advance motor preparation. Because the RT effect is compatible not only with a serial but also with a parallel account, the diagnostic value of RTs in the current task seems to be highly questionable (for a comprehensive discussion of RT effects in serial and parallel models see Townsend, 1990).

LRP. Figure 4A shows the grand mean S-LRPs in go and no-go trials for the blocked and mixed semantic classification mode. This shows the waveforms for trials in which animacy determined the response hand decision. The LRP onset latency for go trials was significantly faster in the blocked- as compared with the mixed semantic classification mode ( $M=432 \mathrm{~ms}$ vs. $M=728$ ms, respectively), $t(23)=4.1, p<.01$, one-tailed. Clearly, the semantic task mixing procedure strongly affected the duration of the animacy classification, as predicted by both serial and parallel models.

\section{A}

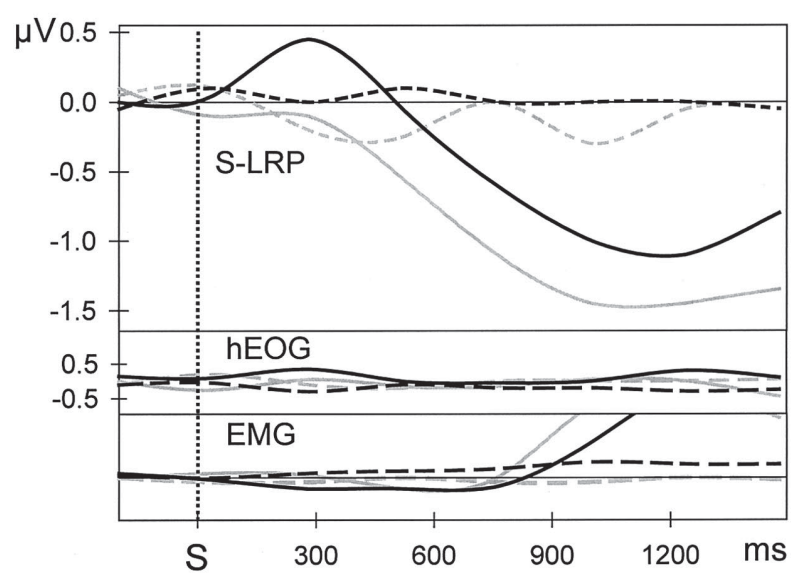

B

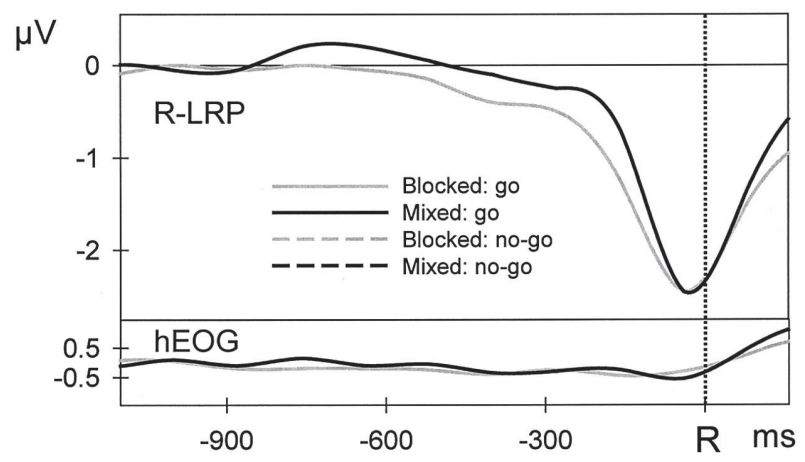

Figure 4. A: Grand mean stimulus-locked lateralized readiness potentials (S-LRPs) for the blocked and mixed semantic classification mode in go and no-go trials. B: Grand mean response-locked LRPs (R-LRPs) for the blocked and mixed semantic classification mode in go trials. The horizontal electrooculogram (hEOG) and ectromyogram (EMG) were computed analogously. $\mathrm{S}=$ stimulus; $\mathrm{R}=$ response.
For no-go trials, an initial LRP was evident only in the blocked semantic classification mode in which the animacy feature could be extracted comparatively fast. Here, the no-go LRP deviated significantly from zero between 440 and $620 \mathrm{~ms}$ poststimulus onset, $t \mathrm{~s}(23)<-2.0, p<.05$. This is a replication of one of the basic results reported by van Turennout et al. (1997) and Schmitt et al. (2000), showing that the animacy information is available earlier than name phonology. However, no such initial LRP was evident in the mixed semantic classification mode. This differential occurrence of the no-go LRP as a function of semantic processing speed is consistent with parallel models only. According to serial models, retrieval of the animacy feature should precede phonological encoding regardless of its duration, leading to a no-go LRP in both conditions. This is not what we observed. Furthermore, the selective occurrence of a no-go LRP in the blocked condition supports the interpretation of the RT results that advance response preparation occurred in the blocked condition only, leading to faster responses. An alternative explanation for the absence of a no-go LRP in the mixed condition could be that the mixing procedure resulted in a general slowing of performance that obliterated advance motor preparation in the mixed condition. Semantic task mixing costs may have altered the task load in the two-choice go/no-go situation such that resources are not sufficient for advance motor preparation. However, in contrast with such an explanation, the preexperiments showed that task mixing at the semantic level did not result in a general slowing of performance. Semantic classification latencies were affected by task mixing but manual name classifications or overt naming latency were not. Additionally, as we discuss below, this alternative explanation is difficult to reconcile with the effects of task mix on the R-LRP.

The R-LRP waveforms for the two experimental conditions are presented in Figure 4B. As can be seen in this figure, for the R-LRP the effect of semantic task mix was reversed; that is, the interval between LRP onset and the response was longer in the blocked as compared with the (slower) mixed classification mode ( $M=-256 \mathrm{~ms}$ vs. $M=-156 \mathrm{~ms}$, respectively), $t(23)=3.8, p<$ .01 , one-tailed. It is important for one to keep in mind that this interval covers the time course of processes between response activation and execution. Clearly, the period between semantic and phonological retrieval was directly affected by the duration of semantic processing. This result is consistent with parallel models because in these models the interval between the completions of semantic and phonological encoding varies as a function of the relative timing of these processes. In contrast, serial models cannot account for this effect. According to serial models, phonological encoding can start only after semantic feature retrieval, and therefore the R-LRP latency should not be affected by the semantic task mixing. It is important to note that the shorter interval between LRP onset and the response in the mixed classification mode cannot be explained in terms of mixing costs, enhanced task load, or a general slowing of performance. This would require the awkward assumption that mixing costs induce faster processing between response activation and execution.

N200. The N200 component, presumably related to response inhibition, is presented in Figure 5. It is interesting but unexpected that there was a slight trend for a later peak latency of the N200 in the blocked relative to the mixed classification mode $(M=552 \mathrm{~ms}$ vs. $M=488 \mathrm{~ms}$, respectively), $t(23)=1.9, p=.07$. Note that neither serial nor parallel models predicted this N200 time course. 


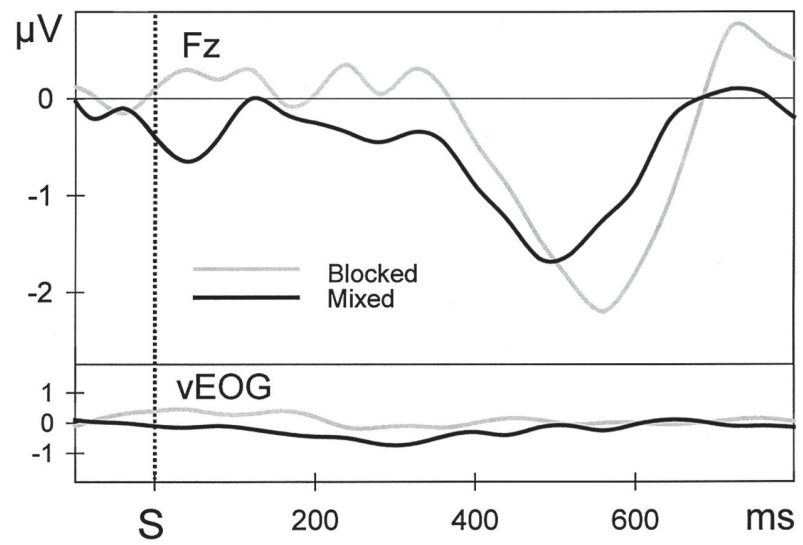

Figure 5. Grand mean N200 effects (difference waves between no-goand go trials) for the blocked and mixed semantic classification mode. The vertical electrooculogram (vEOG) was computed analogously. S = stimulus.

According to serial models, response inhibition is contingent on the extraction of phonological information, which itself depends on the completion of semantic processing. Therefore, serial models predict faster N200 peak latencies for the blocked classification mode than for the mixed classification mode, which is in contrast to the observed effect. According to parallel models, the N200 peak latency should not be affected by semantic task mix. However, it may be the case that given a parallel organization, semantic processing in the mixed classification mode is even slower than word-form encoding, resulting in the observed N200 effect. Yet, this is a post hoc assumption that must be investigated more directly in further experiments.

Alternatively, one could argue that because in the mixed task condition more semantic features are activated, the overall level of semantic activation will be higher than in the blocked task condition. Under the assumption of automatic spreading of activation between processing levels, an increase in activity at the semantic level leads to an increased flow of activation to the lemma level. As a result, the target lemma receives more input from the semantic level and can therefore be selected faster. In turn, its phonological form can be encoded earlier, which leads to earlier response inhibition on no-go trials in the mixed task condition. Thus, according to this idea, although semantic task mixing leads to a delay in the selection of the appropriate semantic feature, the higher level of activity at the semantic level speeds up processing at later, lexical processing levels. Although an interesting possibility, further research is required to test the validity of this hypothesis.

\section{Conclusion}

Taken together, most of the observed effects of semantic task mix in a two-choice go/no-go task are in line with the predictions derived from parallel models and in contrast to what should have been observed according to serial models. First, early response activation on no-go trials was present only in the blocked semantic classification mode. The presence of a no-go LRP replicates earlier findings showing that animacy is retrieved faster than name pho- nology. Critically however, and in contrast to serial models, there was no sign of early response activation in the mixed classification mode in which retrieval latency of the animacy feature was extended. If phonological encoding was mediated by this semantic feature, a no-go LRP should have been present regardless of the duration of semantic feature extraction. Second, the R-LRP onset latency decreased in the mixed as compared with the blocked condition, showing that the interval between the terminations of semantic and phonological encoding varies as a function of the relative timing between these two processes. This result is consistent with parallel models only. Third, the N200 peak latency, an upper estimate of the time needed for the go/no-go decision, tended to be shorter in the mixed classification mode than it was in the blocked classification mode. This is clearly at odds with serial models, which predict the reverse effect. This N200 effect was also not expected from a parallel point of view. However, as explained above, parallel models could easily account for such a result because they allow for a simultaneous activation of semantic and phonological features.

Before turning to theoretical implications of the present results, we discuss two potential caveats, both of which relate to the current, somewhat artificial experimental design. First, consider that semantic task mixing affected the choice decision on the basis of semantic information rather than the actual duration of semantic feature retrieval. In this case the LRP results would reflect differences in response selection latencies between the blocked and mixed classification mode, which should clearly not affect the beginning of phonological encoding. Thus, in this scenario our results would be induced by a task artifact, whereas semantic and phonological processes might be arranged in a strictly serial way. However, the preexperiment provides a strong argument against this alternative. If task mixing affected response selection without altering the time course of semantic feature retrieval, a similar effect should be observed regardless of whether the binary classification is based on a semantic or phonological attribute. This is in contrast to what we observed. Semantic task mixing yielded an effect only on the semantic classification but not on the name classification. We can thus conclude that our manipulation successfully affected the retrieval duration of semantic features.

Second, at a more general level one might ask how the binary semantic classification used in the current experiment is related to semantic activation during normal speech production. Or, in other words, could the retrieval of semantic information for lemma selection be reflected in the categorization latencies for the critical feature? The underlying assumption here is that the S-LRP provides an upper estimate of semantic retrieval time plus additional processes associated with the choice response, such as response selection. Because the results from the preexperiment support the claim that the latter processes do not differ significantly between conditions, we are confident that the present LRP effects reflect semantic processing.

Now, given that our design taps into semantic processing, one might argue that the semantic information retrieved to classify a picture as animal or object or, likewise, the phonological information retrieved to make a response decision, differs from the semantic or phonological information involved in speech production. To our knowledge the empirical evidence supporting such a disjunction of semantic and phonological information into multiple redundant stores, each of which is accessible only for particular 
tasks (e.g., animacy information retrieved for choice responses and animacy information retrieved during picture naming), is particularly scant. Although this account lacks supporting evidence, it remains an option that requires further research. In the most restricted case our results show that animacy information-as recruited for the choice response - can be retrieved in parallel to phonological encoding.

Despite the potential criticism discussed above, the use of ERPs in the study of speech production has clear advantages. Behavioral data such as RTs and accuracy are singular end products of the mental operations involved in speech production. Because the events of interest (here semantic and phonological encoding) typically take place before overt articulation, these measures provide only limited information about the organization of the intervening processes. For example, a distinction between a serial or parallel organization on the basis of RTs can become highly problematic. Thus, ERPs can provide valuable additional information about the timing and interrelationship of covered mental operations during speaking and may therefore be used as a complement to behavioral data as dependent measures. Because the current LRP paradigm is not restricted to specific types of information it can, for example, also be used to examine the relation of syntactic and phonological retrieval in further detail. It is important to note that the current results demonstrate that early availability of a semantic feature in a two-choice go/no-go task as observed in the current and several other studies cannot be taken as conclusive evidence for a serial arrangement of semantic and phonological encoding.

In summary, the current experiment was designed to directly examine whether retrieval of the semantic feature animacy mediates phonological encoding in picture naming. We showed that the beginning of name retrieval was not affected by the duration of semantic processing, an observation that is in contrast with strictly serial models and in line with parallel models. This means that lemma retrieval and word-form encoding do not necessarily depend on the retrieval of predefined semantic attributes. Instead, the present results indicate that in object naming, the word form can be encoded independent of concrete semantic features. This claim appears to be in sharp contrast to the widely shared assumption of a serial ordering of semantic, syntactic, and phonological encoding processes. However, as described in the introduction, parallel retrieval of semantic features and the word form remains a theoretical option. In the model of lexical retrieval proposed by Levelt et al. (1999), ${ }^{1}$ the activation of an abstract concept node (e.g., DOG) by input from different modalities (such as the picture of a dog, a barking dog or, alternatively, the semantic feature ANIMAL) is sufficient for lexical access. Because in this model lemmas are defined as representing syntactic — but not semantic properties of the utterance, the retrieval of any semantic feature that contributes to the meaning of the object name is optional. In other words, the activation of an abstract concept node is the minimal necessary and sufficient prerequisite for lexical access in object naming.

Our results are consistent with the general assumption in speech production models that activity flows from concepts to lemmas to sounds. However, they also indicate that information retrieval at these levels is not strictly ordered in time and that semantic processing before lemma selection and word-form encoding are initiated can be lean. This implies that the exact temporal and functional profile of information retrieval varies as a function of context and task demands, suggesting a highly flexible architecture of the production system.

\footnotetext{
${ }^{1}$ When referring to this model as an example for parallel processing we might introduce some confusion because this model is typically referred to as serial discrete. However, the two terms allude to different aspects of the model architecture. It is serial discrete regarding the information transmission from lemmas to word forms. The term parallel refers to the retrieval of semantic features on the one hand and lemma selection and word-form encoding on the other.
}

\section{References}

Abdel Rahman, R., \& Sommer, W. (2003). Does phonological encoding in speech production always follow the retrieval of semantic knowledge? Electrophysiological evidence for parallel processing. Cognitive Brain Research, 16, 372-382.

Bock, K. (1996). Language production: Methods and methodologies. Psychonomic Bulletin \& Review, 3, 395-421.

Coles, M. G. H. (1989). Modern mind-brain reading: Psychophysiology, physiology and cognition. Psychophysiology, 26, 251-269.

Coles, M. G. H., \& Gratton, G. (1986). Cognitive psychophysiology and the study of states and processes. In G. R. J. Hockey, A. W. K. Gaillard, \& M. G. H. Coles (Eds.), Energetics and human information processing (pp. 409-424). Dordrecht, the Netherlands: Martinus Nijhof.

Collins, A. M., \& Loftus, E. F. (1975). A spreading-activation theory of semantic processing. Psychological Review, 82, 407-428.

De Jong, R., Wierda, M., Mulder, G., \& Mulder, L. J. M. (1988). Use of partial stimulus information in response processing. Journal of Experimental Psychology: Human Perception and Performance, 14, 682-692.

Dell, G. S. (1986). A spreading-activation model of retrieval in sentence production. Psychological Review, 93, 283-321.

Dell, G. S., \& O'Seaghdha, P. G. (1992). Stages of lexical access in language production. Cognition, 42, 287-314.

Eimer, M., \& Coles, M. G. H. (2003). The lateralized readiness potential. In M. Jahanshahi \& M. Hallett (Eds.), The Bereitschaftspotential: In Honour of Professors Deecke and Kornhuber. New York: Kluwer Academic Plenum, 229-248.

Fodor, J. A. (1976). The language of thought. Cambridge, MA: Harvard University Press.

Jasper, H. H. (1958). The ten-twenty electrode system of the international federation. Electroencephalography and Clinical Neurophysiology, 10, 371-375.

Jescheniak, J. D., \& Schriefers, H. (1998). Discrete serial versus cascaded processing in lexical access in speech production: Further evidence from the coactivation of near-synonyms. Journal of Experimental Psychology: Learning, Memory, and Cognition, 24, 1256-1274.

Jodo, E., \& Kayama, Y. (1992). Relation of a negative ERP component to response inhibition in a Go/No-go task. Electroencephalography and Clinical Neurophysiology, 82, 477-482.

Kornhuber, H. H., \& Deecke, L. (1965). Hirnpotentialänderungen bei Willkürbewegungen und passiven Bewegungen des Menschen: Bereitschaftspotential und reafferente Potentiale [Brain potential changes for voluntary and passive movements in humans: Readiness potential and afferent potentials]. Pflügers Archiv für die Gesamte Physiologie, 248, $1-17$.

Kutas, M., \& Donchin, E. (1974, November). Studies of squeezing: handedness, responding hand, response force and asymmetry of readiness potential. Science, 186, 545-547.

Kutas, M., \& Donchin, E. (1980). Preparation to respond as manifested by movement-related brain potentials. Brain Research, 202, 95-115.

Levelt, W. J. M. (1989). Speaking: From intention to articulation. Cambridge, MA: MIT Press. 
Levelt, W. J. M., Roelofs, A., \& Meyer, A. S. (1999). A theory of lexical access in speech production. Behavioral and Brain Sciences, 22, 1-75.

Lippold, O. C. J. (1967). Electromyography. In P. H. Venables \& I. Martin (Eds.), A manual of psychophysiological methods (pp. 245-298). New York: Elsevier.

Los, S. A. (1996). On the origin of mixing costs: Exploring information processing in pure and mixed blocks of trials. Acta Psychologica, 94, $145-188$.

Los, S. A. (1999a). Identifying stimuli of different perceptual categories in mixed blocks of trials: Evidence for cost in switching between computational processes. Journal of Experimental Psychology: Human Perception and Performance, 25, 3-23.

Los, S. A. (1999b). Identifying stimuli of different perceptual categories in pure and mixed blocks of trials: Evidence for stimulus-driven switch costs. Acta Psychologica, 103, 173-205.

Low, K. A., \& Miller, J. (1999). The usefulness of partial information: Effects of go probability in the choice/nogo task. Psychophysiology, 36, $288-297$.

Miller, J., \& Hackley, S. A. (1992). Electrophysiological evidence for temporal overlap among contingent mental processes. Journal of Experimental Psychology: General, 121, 195-209.

Miller, J., Patterson, T., \& Ulrich, R. (1998). Jackknife-based method for measuring LRP onset latency differences. Psychophysiology, 35, 99115 .

Nieuwenhuis, S., Yeung, N., van den Wildenberg, W., \& Ridderinkhof, K. R. (in press). Electrophysiological correlates of anterior cingulate function in a go/nogo task: Effects of response conflict and trial-type frequency. Cognitive, Affective, and Behavioral Neuroscience.

Oldfield, R. C. (1971). The assessment and analysis of handedness: The Edinburgh inventory. Neuropsychologia, 9, 97-113.

Osman, A., Bashore, T. R., Coles, M. G. H., Donchin, E., \& Meyer, D. E. (1992). On the transmission of partial information: Inferences from movement-related brain potentials. Journal of Experimental Psychology: Human Perception and Performance, 18, 217-232.

Peterson, R. R., \& Savoy, P. (1998). Lexical selection and phonological encoding during language production: Evidence for cascaded processing. Journal of Experimental Psychology: Learning, Memory, and Cognition, 24, 539-557.

Roelofs, A. (1992). A spreading-activation theory of lemma retrieval in speaking. Cognition, 42, 107-142.
Roelofs, A. (1997). A case of nondecomposition in conceptually driven word retrieval. Journal of Psycholinguistic Research, 26, 33-67.

Schmitt, B. M., Münte, T. F., \& Kutas, M. (2000). Electrophysiological estimates of the time course of semantic and phonological encoding during implicit picture naming. Psychophysiology, 37, 473-484.

Schmitt, B. M. , Rodriguez-Fornells, A., Kutas, M., \& Münte, T. F. (2001). Electrophysiological estimates of semantic and syntactic information access during tacit picture naming and listening to words. Neuroscience Research, 41, 293-298.

Schmitt, B. M., Schilz, K., Zaake, W., Kutas, M., \& Münte, T. F. (2001). An electrophysiological analysis of the time course of conceptual and syntactic encoding during tacit picture naming. Journal of Cognitive Neuroscience, 13, 510-522.

Simson, R., Vaughan, H. G., \& Ritter, W. (1977). The scalp topography of potentials in auditory and visual Go/NoGo tasks. Electroencephalography and Clinical Neurophysiology, 43, 864-875.

Smid, H. G. O. M., Mulder, G., \& Mulder, L. J. M. (1987). The continuous flow model revisited: Perceptual and central motor aspects. In R. Johnson Jr., J. W. Rohrbaugh, \& R. Parasuraman (Eds.), Current trends in event-related potential research (EEG Suppl. 40, pp. 270-278). Amsterdam: Elsevier.

Smid, H. G. O. M., Mulder, G., Mulder, L. J. M., \& Brands, G. J. (1992). A psychophysiological study of the use of partial information in stimulus-response translation. Journal of Experimental Psychology: Human Perception and Performance, 18, 1101-1119.

Thorpe, S., Fize, D., \& Marlot, C. (1996, June). Speed of processing in the human visual system. Nature, 381, 520-522.

Townsend, J. T. (1990). Serial vs. parallel processing: Sometimes they look like tweedledum and tweedledee but they can (and should) be distinguished. Psychological Science, 1, 46-54.

van Turennout, M., Hagoort, P., \& Brown, C. M. (1997). Electrophysiological evidence on the time course of semantic and phonological processes in speech production. Journal of Experimental Psychology: Learning, Memory, and Cognition, 23, 787-806.

van Turennout, M., Hagoort, P., \& Brown, C. M. (1998, April). Brain activity during speaking: From syntax to phonology in 40 milliseconds. Science, 280, 572-574.

Vaughan, H. G., Jr., Costa, L. D., \& Ritter, W. (1968). Topography of the human motor potential. Electroencephalography and Clinical Neurophysiology, 25, 1-10. 


\section{Appendix}

Picture Stimuli Used in the Experiments

\begin{tabular}{|c|c|c|c|}
\hline \multicolumn{2}{|c|}{ Inside the water } & \multicolumn{2}{|c|}{ Outside the water } \\
\hline Vowel & Consonant & Vowel & Consonant \\
\hline \multicolumn{4}{|c|}{ Animal } \\
\hline Dolfijn [dolphin] & Zwaan [swan] & Varken [pig] & Spin [spider] \\
\hline Kikker [frog] & Krokodil [crocodile] & Muis [mouse] & Olifant [elephant] \\
\hline Eend [duck] & Krab $[\mathrm{crab}]$ & Hond $[\mathrm{dog}]$ & Vlinder [butterfly] \\
\hline Haai [shark] & Flamingo [flamingo] & Vos $[$ fox $]$ & Ezel [donkey] \\
\hline Zeepaard [seahorse] & Kwal [jellyfish] & Haan [rooster] & Schaap [sheep] \\
\hline Garnaal [prawn] & Inctvis [squid] & Haas [hare] & Vlieg [fly] \\
\hline Seester [starfish] & Kreeft [cancer] & Uil [owl] & Egel [hedgehog] \\
\hline \multicolumn{4}{|c|}{ Object } \\
\hline Duikbril [diver eyeglasses] & Snorkel [snorkel] & Hamer [hammer] & Schaar [scissors] \\
\hline Kano [canoe] & Flippers [fins] & Boek [book] & Schoen [shoe] \\
\hline Haak [fishhook] & Anker [anchor] & Auto [car] & Tractor [tractor] \\
\hline Boei [buoy] & Schelp [shell] & Koffer [trunk] & Bril [glasses] \\
\hline Zeilboot [sailboat] & Onderzeer [submarine] & Camera [camera] & Stempel [stamp] \\
\hline Gondel [gondola] & Opblaasboot [dinghy] & Kaars [candle] & Vliegtuig [airplane] \\
\hline
\end{tabular}

Note. Dutch words are shown with English equivalents in brackets. Pictures assigned to filler trials are shown in bold.

Received August 6, 2002 Revision received January 19, 2003

Accepted January 20, 2003

\section{New Editor Appointed for Contemporary Psychology: APA Review of Books, 2005-2010}

The Publications and Communications Board of the American Psychological Association announces the appointment of Danny Wedding (Missouri Institute of Mental Health) as editor of Contemporary Psychology: APA Review of Books, for a 6-year term beginning in 2005. The current editor, Robert J. Sternberg (Yale University), will continue as editor through 2004.

All reviews are written by invitation only, and neither the current editor nor the incoming editor receives books directly from publishers for consideration. Publishers should continue to send three copies of books for review consideration, along with any notices of publication, to PsycINFO Services Department, APA, Attn: Contemporary Psychology: APA Review of Books Processing, P.O. Box 91600, Washington, DC 20090-1600 or (for UPS shipments) 750 First Street, NE, Washington, DC 20002-4242. 